\title{
Designing a spatial pattern to rebalance the orien- tation of development and protection in Wuhan
}

\author{
JIN Gui ${ }^{1}$, SHI Xin ${ }^{2},{ }^{*} H E$ Dawei ${ }^{2}$, GUO Baishu², LI Zhaohua², SHI Xianbin ${ }^{3}$ \\ 1. College of Urban and Environmental Sciences, Central China Normal University, Wuhan 430079, China; \\ 2. Faculty of Resources and Environmental Science, Hubei University, Wuhan 430062, China; \\ 3. Hubei Geological Survey Institute, Wuhan 430030, China
}

\begin{abstract}
Patterns of spatial development and protection form a basic category of geoscience, and redesigning them is a popular subject of research in regional sustainable development that is important for ecological civilization construction. The authors here report a case study of Wuhan city using the circuit theory model and minimum cumulative resistance (MCR) model to rebalance its spatial protection and development. The results show the following: (1) Using the density of the gross domestic product (GDP), density of population, rate of urbanization, and access to transportation as evaluation indicators, seven core areas of development in Wuhan were identified, accounting for $59 \%$ of the total number of streets, that exhibited a "circular-satellite" spatial structure. (2) According to the importance of ecosystem services, ecological sensitivity, land use type, and slope of the terrain, the resistance surface of spatial development in Wuhan had a stereoscopic spatial form of an "inverted pyramid," with high surroundings and a low center. The area of low resistance accounted for $6.64 \%$ of the total area of Wuhan. (3) Based on coupling analysis using the MCR and spatial morphological characteristics of current, nine axes of spatial development with a total area of 427.27 $\mathrm{km}^{2}$ and eight key strategic points with a total area of $40.02 \mathrm{~km}^{2}$ were identified. Streets that were prioritized for development accounted for $9.63 \%$ of Wuhan's total area. (4) By combining the characterization of the development axis with the structure of the three-level core area, we extracted the structure of spatial development of "one heart, two wings, and three belts" in Wuhan. The research framework and empirical results can provide scientific guidance for the urban spatial layout, the development of regional linkages, and ecological environmental protection in China.
\end{abstract}

Keywords: spatial development and protection; circuit theory model; minimum cumulative resistance model; core area; development axis; Wuhan

Received: 2019-04-25 Accepted: 2019-12-29

Foundation: National Natural Science Foundation of China, No.71974070, No.41501593; Social Sciences Foundation of Ministry of Education of China, No.19YJCZH068, Scientific Research Project of Hubei Provincial Natural Resources Department, No.ZRZY2019KY08

Author: Jin Gui (1986-), PhD and Associate Professor, specialized in land resource evaluation and national land management. E-mail: jingui@igsnrr.ac.cn

"Corresponding author: He Dawei, E-mail: Hedw09@163.com 


\section{Introduction}

The goals of constantly optimizing the pattern of national spatial development and constructing an ecological civilization were proposed at the 19th National Congress of the Communist Party of China (CPC) in 2017. The document stated that the state had upgraded the optimization of patterns of spatial development to the national strategic level, and considered it an important foundation for ecological civilization construction (Huang et al., 2015). Through spatial planning and controlling human activities to promote the coordination of population distribution, economic layout and resources and environmental carrying capacity - in other words, the guidelines of the blueprint - have become crucial for spatial optimization. The difficulty of "sticking to a single blueprint until the end" lies in the scientific and quantitative depictions of the structure of spatial development (Fan, 2018). Most scholars in China and abroad have studied quantitative depictions following two research paradigms. From the perspective of development, they have developed "point-axis-area" development projects to promote economic growth by characterizing regional functions and spatial structures under interregional interactions (Zhou et al., 2015; Bradburd et al., 2016). This also serves the interests of planning for national economic development and implementing the relevant policies at the spatiotemporal scale, and promotes orderly urban spatial expansion (Todes, 2017; Chen et al, 2018). From the perspective of protection, researchers have assessed the spatiotemporal pattern, process, and mechanism of regional landscape ecology for ecological conservation, and have highlighted the patterns of ecological security as a means of alleviating the contradiction between ecological protection and economic development to protect the ecological space (Ferretti and Pomarico, 2013; Correa Ayram et al., 2016; Nunes et al., 2019). Based on the "point-axis" theoretical framework, the central points, axes of development, and core areas of land space were measured and portrayed. The most widely used methods are spatial clustering and kernel density analysis (Vasanen, 2012; Veneri, 2013; Yu et al., 2014). Another method involves identifying the ecological sources, corridors, and patches at all levels using theories on the patterns of ecological security of land space to develop systematic patterns. Commonly used methods for the network analysis of minimum paths include redundancy analysis, circuit theory, random walk theory, minimum cumulative resistance (MCR), and the cost distance model (Blazquez-Cabrera et al., 2016; Luo et al., 2018; Li et al., 2019). Regardless of the perspectives of development or protection, the purpose of the existing research is to use a single goal as the direction of space management (Peng et al., 2015; Jin et al., 2019), ignoring the mutual competition and synergy within various land spaces. Few studies have incorporated agricultural space into the research framework of competition for land use and the relevant trade-offs.

In the backdrop of promoting well-coordinated environmental conservation and avoiding excessive development, it is important to form patterns for the development and protection of space to use it efficiently, create a livable space, and ensure that the ecological space is unspoiled. Based on a combination of point-axis theory and the theory of patterns of ecological safety, we selected Wuhan as study area and used the random walk theory and MCR to implement function recognition. Quantitative description of the axis of development and a comprehensive expression of the spatial form of the natural-human system are also provided. Scientific patterns of spatial development and protection for Wuhan can provide reference for decisions on the regional ecological civilization construction and the management of natural resources. 


\section{Study area}

Wuhan is located in central China, in the middle reaches of the Yangtze River, and is an important node city for the Belt and Road Initiative (Figure 1). By the end of 2017, Wuhan's population and GDP accounted for $18.46 \%$ and $36.72 \%$, respectively, of those of Hubei province, and its urban built-up area was $585.61 \mathrm{~km}^{2}$. The expansion of the city is based on the natural pattern formed by such elements of the ecological landscape as mountains, rivers, and lakes, and serves as a corridor for the urban landscape and pattern of economic development (Liu et al., 2015; Yang et al., 2019). Large scale, low density, and imbalance are typical characteristics of the Yangtze River Economic Belt, and have hindered the sustainable development of Wuhan (Jin et al., 2018). Therefore, it is important to form a "point-axis-area" spatial structure that can help reap the comprehensive benefits of its geographical location and natural ecosystem according to the demands of human activities. A research framework for Wuhan that considers the patterns of spatial development and protection during construction can provide reference for decisions on regional spatial governance and further urbanization as well as guidance for the urban spatial layout, development of regional linkages, and environmental protection of the Yangtze River Economic Belt. It is also significant for theoretical exploration and practical guidance for ecological civilization construction of in China.

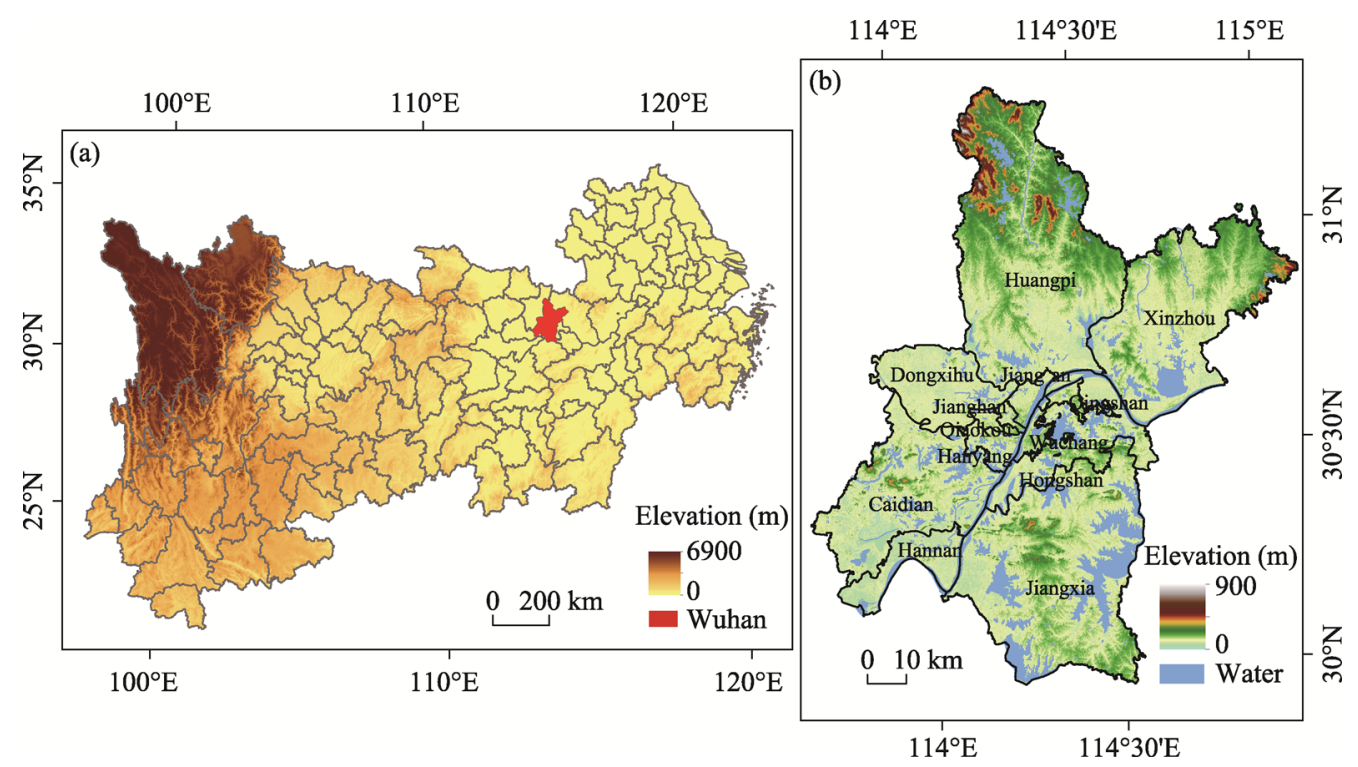

Figure 1 Location of Wuhan within the Yangtze River Economic Belt (a), and administrative divisions of the city (b)

\section{Data and methods}

\subsection{Data sources and processing}

The research data included natural environmental data to characterize the indigenous ecosystem and socioeconomic data to reflect the appropriate needs of the relevant human activities. The natural environmental data included the following: (1) Data from meteorological stations of the National Meteorological Information Center (http://data.cma.cn/), including 
temperature and rainfall, were used to measure ecological sensitivity and assess ecological vulnerability. (2) Data on soil, from the 1:1,000,000 Soil Database of China (http://vdb3. soil.csdb.cn/), included soil texture, granules, and hydrology to estimate soil seepage. (3) Data on the land use type and elevation, with a spatial resolution of $30 \times 30 \mathrm{~m}$, were obtained from the Resources and Environmental Data Cloud Platform (http://www.resdc.cn) to extract the resistance surface (degree of inhibition of spatial development by each unit). The land use data were classified into built-up land, cultivated land, forest land, grassland, water area, and unused land according to the taxonomy system of the Chinese Academy of Sciences. The socioeconomic data included the following: (4) The population, GDP, and other data on townships in Wuhan were used to identify the core area. The data were from the Wuhan Statistical Yearbook, the Wuhan National Economic and Social Development Statistical Bulletin, and each district's statistical yearbook. (5) Road network data, used to calculate access for traffic, were from the National Geomatics Center of China (http://www. ngcc.cn/). Considering the availability of data, timeliness of research, and the generality of the plan's base year, we focused on 2018 for the study, and all grids of the raster data were resampled to $100 \mathrm{~m} \times 100 \mathrm{~m}$.

\subsection{Minimum cumulative resistance}

On the basis of geography theory and geographic information, the MCR has been proposed to quantify the cost or the minimum resistance of the object from the source through different patches to the destination (Blazquez-Cabrera et al., 2016). It is expressed as follows:

$$
M C R=f_{\min } \sum_{j=n}^{i=m} D_{i j} \times R_{i}
$$

where $M C R$ denotes the minimum cumulative resistance, $D_{i j}$ represents the spatial distance of spatial elements flowing from core area $j$ to the specified unit $i, R_{i}$ represents the coefficient of resistance of spatial unit $i$ to the elements, and $f$ represents the positive correlation function between the minimum cumulative resistance and the process of spatial expansion. Based on this model, the grid matrix can be used to construct the resistance matrix in combination with the spatial position, following which the surface feature of resistance can be simulated, that is, the resistance surface of the spatial pattern can be extracted.

The spatial characteristics of the elements flow revealed by the MCR reflect the possibility and trend of the spatial structure, and are used to measure the change in overall connectivity by adjusting channel width (Leonard et al., 2017). Due to differences in natural characteristics among locations, the process of expansion of human activities is resisted to varying degrees. In particular, national nature reserves with good ecological risk prevention capabilities are imposed on stringent demands on the expansion of construction land and urban space (Jin et al., 2019). The connectivity and similarity between the spatial unit and the source unit are determined by the magnitude of the MCR. The potential spatial paths of elements flowing from source to destination, that is, the plaque connection channel, can then be extracted by lateral comparative analysis.

\subsection{Circuit theory model}

The circuit theory model links the state of motion of an object to resistance to it based on random walk to yield the trajectory of spatial motion (Leonard et al., 2017). Objects in random walk are in an unpredictable state, but the trajectory of charge in the circuit can be sim- 
simulated by effective resistance and voltage (Roopnarine et al., 1999; Blatti and Sinha, 2016). Thus, the circuit theory model can be used to explore the laws of flow of the spatial elements. A circuit diagram is a meshed network connected by "resistors" that are similar in principle to the mechanism of formation of the spatial structure (McRae and Beier, 2007). When studying the morphological features of spatial elements, resistance is often expressed by the difficulty with which an object passes through the plaque, and the total resistance between nodes is used to represent the resistance distance. Thus, the effective resistance directly determines the characteristics of connectivity of different spatial nodes.

The basic indicators of the circuit theory model are current $I$, voltage $V$, and resistance distance $D_{R}$, where the latter is the decisive factor in the formation of connections during spatial development, and its value is determined by the size of the plaque and number of paths. When two nodes can be connected through multiple paths with low resistance, the resistance distance is small; when they can be connected through few paths with high resistance, the resistance distance is large. The current, voltage, and resistance distance are calculated as follows (McRae and Beier, 2007; Thulasiraman et al., 2019):

(1) Current: When the spatial element flows from node $A$ to node $B$, the current $i_{x y}$ of adjacent nodes $x$ and $y$ is the expected value of the net number of times it flows through the directed edge $x y$ during the random walk of the element from $A$ to $B$, as follows:

$$
i_{x y}=\left(v_{x}-v_{y}\right) C_{x y}=\left(\frac{u_{x}}{C_{x}}-\frac{u_{y}}{C_{Y}}\right) C_{x y}=\frac{u_{x} C_{x y}}{C_{x}}-\frac{u_{y} C_{y x}}{C_{\mathrm{y}}}=u_{x} p_{x y}-u_{y} p_{y x}
$$

where $u_{x}=\sum_{y} u_{y} p_{y x}$ represents the average number of times the spatial element reaches state $x$ before reaching node $B, u_{x} p_{x y}$ represents the average number of times a spatial element flows from $x$ to $y, u_{y} p_{y x}$ represents the average number of times a spatial element flows from $y$ to $x$, $i_{x y}$ represents the average net number of spatial elements flowing between $x$ and $y$, and $C_{x y}$ denotes the conductance of $x$ and $y$, which is used to measure the flow capability of spatial elements between nodes, and has the properties $C_{x y}=C_{y x}=1 / R_{x y}, C_{x}=\sum_{y} C_{x y}$, and $C_{y}=\sum_{x} C_{y x} . P_{x y}$ represents the element of the transfer matrix $P, P_{x y}=C_{x y} / C_{x}$, which is used to characterize the spatial pattern (assumed to be $G$ ) formed by points and lines according to the trajectory of random walk that conforms to the Markov chain.

(2) Voltage: When there is a spatial relationship between nodes $A$ and $B$, and $A$ is the starting point of the process of elements flow, the voltage $v_{x}$ of point $x$ indicates the probability that the spatial elements return $A$ from $x$ before returning to $B$, as follows:

$$
\mathrm{v}_{x}=\sum_{y} \frac{c_{x y}}{c_{x}} v_{y}=\sum_{y} p_{x y} v_{y}, \forall x \neq a, b
$$

(3) Resistance distance: The resistance distance between nodes $u$ and $v$ is expressed by $R(u, v)$, that is, the degree of connectivity between nodes $u$ and $v$ in the spatial pattern (Klein and Randić, 1993). The resistance distance is formed by combining the common distance and resistance of fixed resistors in the network. The Kirchhoff index $K f(G)$ can be expressed in the form of the Wiener index (Dobrynin and Kochetova, 1994) as follows:

$$
K f(G)=\sum_{\{u, v\} \subseteq V(G)} R_{G}(u, v)
$$

The resistance distance characterized by the Wiener index is as follows:

$$
D_{R}(G)=\sum_{\{u, v\} \subseteq V(G)}[d(u)+d(v)] \cdot R(u, v)
$$


where $d(u)=d G(u)$ and $d(v)=d G(v)$ denote the number of axes connecting nodes $u$ and $v$ on the spatial network, respectively.

Applying the framework of circuit theory to study regional patterns can improve the understanding of new regional patterns shaped by the flow of spatial elements and provide means for interpreting complex geographical changes. Based on this framework, we propose research ideas at three levels of a system composed of the core area, resistance surface, and axis of development. The urban node network is characterized as a circuit, resistance values are set according to the suitability of the spatial unit, and the connectivity of regional units is measured using the resistance distance (Figure 2a). When resistance was small, the current of the unit voltage was correspondingly large, and the path of transmission of the element through the core regions was gradually formed. Note that when there was a "barrier point" between $\mathrm{A}$ and $\mathrm{B}$ in the spatial pattern, that is, a high-resistance unit, the axis of development through the "barrier point" was directly broken. When there was a "pinch point" (strategic point) in the spatial pattern as major node, the degree of connectivity directly determined the spatial characteristics of the axis of development (Figure 2b).
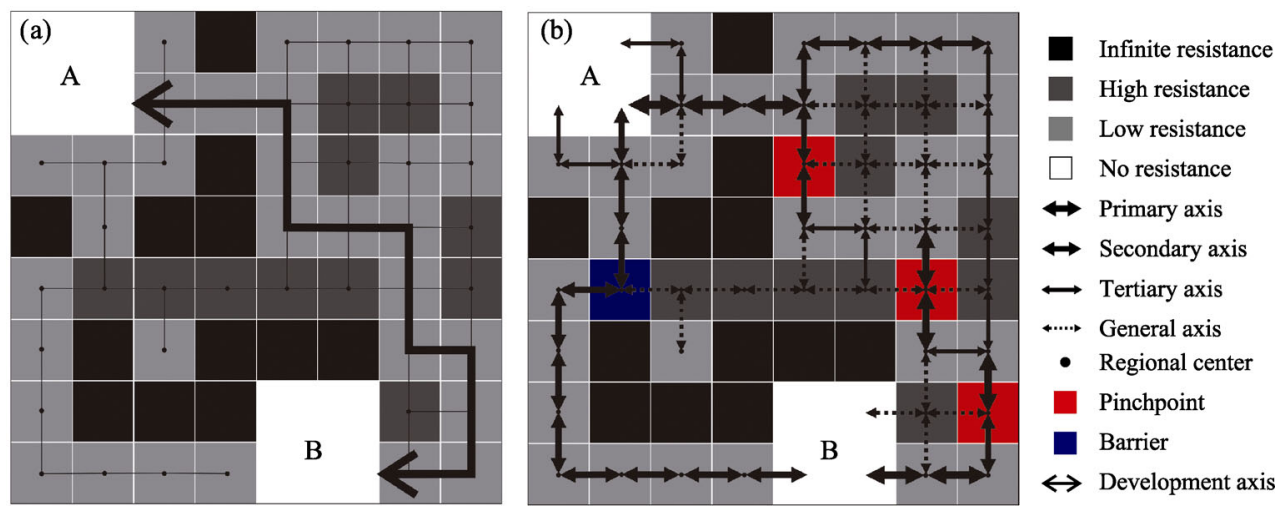

Figure 2 Schematic of circuit theory: resistance surface (a) and spatial pattern (b) (Spear, 2010)

\subsection{Identifying core area and extracting resistance surface}

This study aims to clarify the scientific connotation of the patterns of ecological security identified by the evaluation indicators of the importance of the habitat, its ecological sensitivity, and the connectivity of its landscape (Azaele et al., 2015; Costanza and Terando, 2019). These indicators are then combined to express the characteristics of human behavior and urban expansion, such as the rate of land urbanization, GDP density, population density, and access for traffic (Jin et al., 2017). We assessed the importance and potential of each street of Wuhan in the patterns of spatial development, and streets with remarkable advantages were used as core areas of development. Due to the obstruction of such agricultural and ecological surface features as permanent basic cropland and wetland, it is necessary to first construct the resistance surface to express the characteristics of flow of the spatial elements (Martin et al., 2018). The indigenous characteristics of Wuhan's ecological system as well as its policies for ecological conservation and nature reserves combined with the ecological indicators (i.e., importance of ecosystem services, ecological sensitivity, land use type and terrain slope) were used to extract the resistance surface of spatial development. Considering the relationship between the nature of land and its potential for spatial devel- 
opment, the coefficient of resistance of land use types in ascending order was found to be built-up land, unused land, forest land, grassland, cultivated land, and water area. Using an ensemble algorithm, the characteristic of development value of each spatial unit, that is, its resistance value, was calculated, and the result was a value between 0 and 100 . Value 0 meant that there was no obstacle to the flow of elements, and value 100 meant that the spatial elements could not flow, that is, they could not form a connection channel through the neighboring unit.

\subsection{Characterizing development axis and constructing the geospatial pattern}

Geospatial data were converted into a graph structure and the Laplacian operator was constructed to calculate the effective resistance, current, and voltage. All possible paths were then integrated into the calculation of distance to identify the potential axes of spatial development by an analog simulation of the flow of spatial elements (McRae et al., 2008; Kupfer, 2012). In practice, the axes of development are spatial entities with a striped shape and a certain width, where the basic network structure of spatial development is determined by the attributes of the development axes and nature of the passing spatial unit (Table 1). Due to the differences and the presence of a hierarchy, the core areas exerted different influences on the other territorial units, thus forming a pattern of protection and development with spatial elements at different levels. The identification of strategic points and measurement of centrality are crucial for the analysis of these elements. Strategic points are the key nodes in the development axes that determine regional connectivity (Avon and Bergès, 2016; Peng et al., 2018) and control the flow of elements in space. Centrality is an evaluation index based on current flow that characterizes the ability of elements to be transmitted from the core area to other areas (Carroll et al., 2012). Based on the technical regulations of the circuit theory model and MCR, we measured the current along the least-cost path, analyzed the centrality of the core areas, and identified the strategic points. In the course of the spatial planning, a spatial geographical pattern with efficient elements allocation, orderly spatial structure, and coordinated regional functions was constructed by guiding the spatial elements to the core areas and strategic points.

Table 1 Comparison of physical terms and their geographical significance in circuit theory

\begin{tabular}{ll}
\hline \multicolumn{1}{c}{ Terms } & \multicolumn{1}{c}{ Geographical significance } \\
\hline Current & $\begin{array}{l}\text { Probability of each element flowing through a geographic unit in the pattern of spatial pro- } \\
\text { tection and development } \\
\text { The resistance to development of the spatial unit; the geographical unit with a tendency } \\
\text { toward protection is assigned a high resistance value }\end{array}$ \\
Thesistance & $\begin{array}{l}\text { The probability that the spatial element leaves a node and successfully reaches a specified } \\
\text { node is also the probability that two nodes can be connected through the axis } \\
\text { Resistance distance of different paths between nodes }\end{array}$ \\
Ground & \begin{tabular}{l} 
Endpoint of the spatial development axis \\
\hline
\end{tabular}
\end{tabular}

\section{Results and analysis}

\subsection{Core area and resistance surface}

The core areas were identified by integrating the calculations of population density, GDP density, traffic accessibility, and rate of land urbanization to express the characteristics of spatial differences that were suitable for human activities. We selected $20 \%$ as the key 
threshold for the identification of the core areas (Peng et al., 2018), and extracted seven core areas in Wuhan based on the criterion of spatial continuity and characteristics of spatial heterogeneity. They featured 111 streets, accounting for $59 \%$ of the total number of streets in the city (Figure 3a). The extracted core areas showed a "circular-satellite" distribution centered on core area 4, which was located in Wuhan's central urban area and accounted for $70.31 \%$ of the total area of core areas. It exhibited a horizontal extension of the transition to Dongxihu along the rail, urban trunk road, and other traffic lines. The other core areas distributed in the administrative area of Wuhan were in the form of mosaics, and exhibited a high degree of consistency with the district-level political center because of significant differences in the processes of natural distribution and redistribution of spatial elements in the regions. The administrative center thus had advantages in terms of population, land use, and a transportation network to form and strengthen the spatial characteristics in the context of preferential policies, spatial resource agglomeration, and robust foundations for development in the core areas. As lowlands of policies and resources, the core areas play a leading role in regional development due to their resources endowments, and their strategic support-related position should be clearly defined during spatial planning.
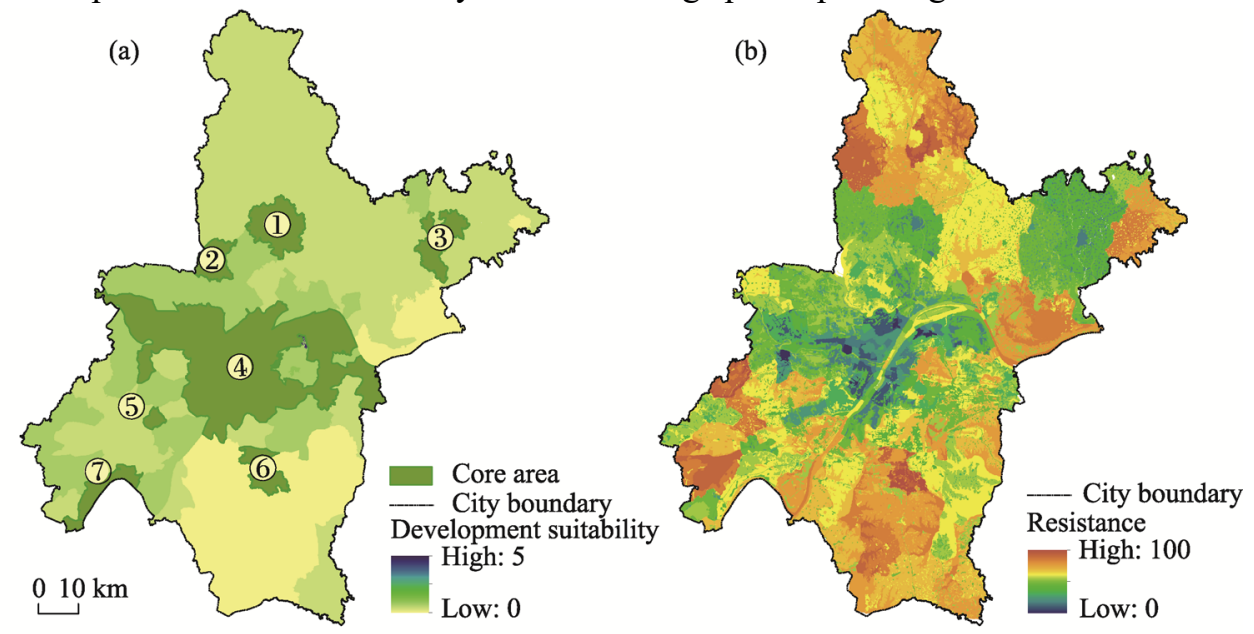

Figure 3 Spatial distribution of core areas (a) and development resistance (b)

The extraction of the resistance surface requires the comprehensive consideration of natural indigenous characteristics and the needs of human ecological security. An index system that considers ecological sensitivity and the importance of ecosystem services based on this consideration can have important scientific and policy-related implications. The global feature of the resistance surface extracted by the ensemble algorithm was the "inverted pyramid" distribution in which the resistance decreased from the periphery to the center (Figure $3 b$ ). The streets that were far from the administrative center were assigned more stringent responsibilities related to ecological security, which is consistent with the scientific understanding of the low ecological service function and high socioeconomic development function of the core areas. High ecological service functions and low socioeconomic development functions were assigned to the resistance surface. Areas with high resistance were mainly distributed in the ecological space with significant ecological benefits, such as the basic agricultural development zone in the center of Huangpi and Xinzhou, and the southwest of Caidian. The ecological function protection areas were in the center of Huangpi, and the east and southwest of Xinzhou. To extract more spatiotemporal features of the 
the east and southwest of Xinzhou. To extract more spatiotemporal features of the resistance surface, 10 and 90 were set as the upper (development tendency) and lower (protection tendency) limits of development resistance, respectively. Areas that had low development resistance values covered an area of $568.77 \mathrm{~km}^{2}$ accounting for $6.64 \%$ of the total area of Wuhan, concentrated in the periphery of the core areas with a flat terrain and high development potential. Areas with high development resistance values covered $1073.37 \mathrm{~km}^{2}$ and accounted for $12.53 \%$ of the city's total area, mainly distributed in the north of Huangpi, southeast of Xinzhou, southwest of Caidian, and in south-central Jiangxia. By comparing resistance values inside the core zone, an average resistance in the core area 5 was calculated to be the smallest, whereas the average resistance in core zone 6 was over 90 .

\subsection{Development axis and geospatial pattern}

Based on the MCR, "linear" channels with a maximum width of $6 \mathrm{~km}$ between the core areas were extracted by the Linkage Mapper as the axis of development to quantitatively describe the spatial characteristics of elements flow (Figure 4a). The results show that, first, the extracted development axes covered an area of $427.27 \mathrm{~km}^{2}$, accounting for $5 \%$ of the total area of Wuhan. Second, the core areas played leading roles in the connectivity of the development axes, where core area 4 was connected with the other core areas by six development axes, and other core areas were connected to one another by only two or three axes. This exacerbated the spatial differences in terms of the intensity of communication. Finally, not all core areas were connected by the development axis, some transit nodes satisfied elements exchanged between regions. For example, core areas 4 and 7 realized interregional communication with core area 6 as transit point. However, only the least-cost path was found to be inadequate to guide the design of Wuhan's spatial planning in practice, and it was thus necessary to refine the channels of spatial development of different widths to supplement the communication and coordination between core areas.
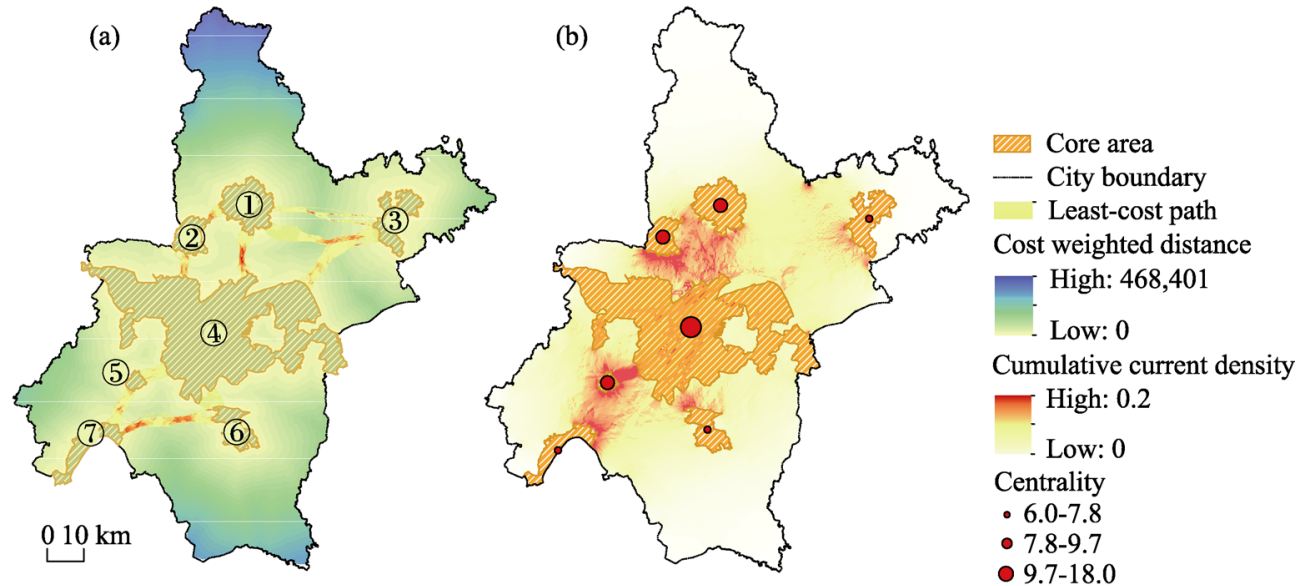

Figure 4 Least-cost path (a) and current density (b) of spatial development

Based on the measured results of the resistance distance between core areas, we used iterative calculation to obtain their current densities, where this reflected the degree of spatial connections of each core area in Wuhan. The resistance distance could identify the potential characteristics of spatial connections under the condition that other factors were uniform to 
quantificationally reflect the important features and spatial structure of the spatial elements. The results show that the resistance distances of core areas 1-2, 1-4, 2-4, 4-5, and 4-6 were all shorter than 10, whereas the resistance distances of core areas 1-7, 2-3, 2-7, 3-5, 3-6, and 3-6 were all longer than 20 . When the resistance distance was longer than 13 , a direct connection could not be formed between the core areas (Table 2). Table 2 shows that the resistance distances between the core areas were prominently different, and this value was affected by the mutual positional relationship and connection of the spatial elements between core areas.

Table 2 Resistance distance between pairwise cores

\begin{tabular}{cccccccc}
\hline \multirow{2}{*}{ Code of core area } & \multicolumn{7}{c}{ Code of core area } \\
\cline { 2 - 7 } & 2 & 3 & 4 & 5 & 6 & 7 \\
\hline 1 & 5.06 & 17.71 & 6.75 & 17.95 & 17.34 & 26.01 \\
2 & & 21.31 & 5 & 15.11 & 15.37 & 23.26 & 37.1 \\
3 & & 16.22 & 29.15 & 27.77 & 13.72 & 12.8 \\
4 & & & 4.87 & & 13.29 & & 19.25 \\
5 & & & & & & & \\
6
\end{tabular}

As a representative indicator of resistance distance and the importance of core areas, current density was used to characterize the spatial correlation between a core area and adjacent core areas. The highest current density in Wuhan was about $1495.27 \mathrm{~km}^{2}$, and appeared as a "center-peripheral" layered sphere in which core areas 1-2-4 and 4-5-7 were the "two peaks" of current density. The high current density around core areas 1 and 2 showed a "planar" spatial distribution, which provides strong support for the flow of spatial elements between core areas. Regions of high current density between core areas 4 and 5 were "linearly" distributed (Figure 4b). Specifically, distant suburban areas, such as Huangpi, Xinzhou, Jiangxia, and Caidian, had low current density and weak regional flows of spatial elements. Central urban areas of Wuhan, such as Wuchang, Hankou, and Hongshan, had higher levels of socioeconomic development and more frequent flow of spatial elements, indicating that current density in these areas was still in the stage of unbalanced growth. By comparing the least-cost path with current density, a spatial inconsistency was observed between the two types of channels. The former identified the path of spatial development quantitatively by the cost of elements flow, and the latter broke through the given state of the connected features, development axes as characterized by it were forward looking, innovative, and complete. Through a combination of the least-cost path and current density, eight strategic points for spatial development in Wuhan with a total area of $40.02 \mathrm{~km}^{2}$ were identified as a measure of connectivity to complement the least-cost distances, and consisted of 10 streets (Figure 4a). The centrality of core areas in Wuhan was divided into three levels. Core area 4 had a higher link centrality score than the others because it was a "hub" for keeping the spatial patterns connected (Figure 4b).

To construct a practical hierarchical system for spatial development and protection in Wuhan, a structure consisting of ordered core regions at three levels was set up. The first level was core area 4 with central urban areas as the main body. There were six core areas at the second level, and those at the third level were strategic points that influenced the development axes. Core regions at the third level (strategic point) were invisible growth points of 
spatial development based on the axis of development characterized by the least-cost path, and coupled with current density for the recognition of spatial morphological features (Figure 5). As potential growth points for spatial development, the third level of core regions should focus on developing the new organizational model and forming new industrial space.

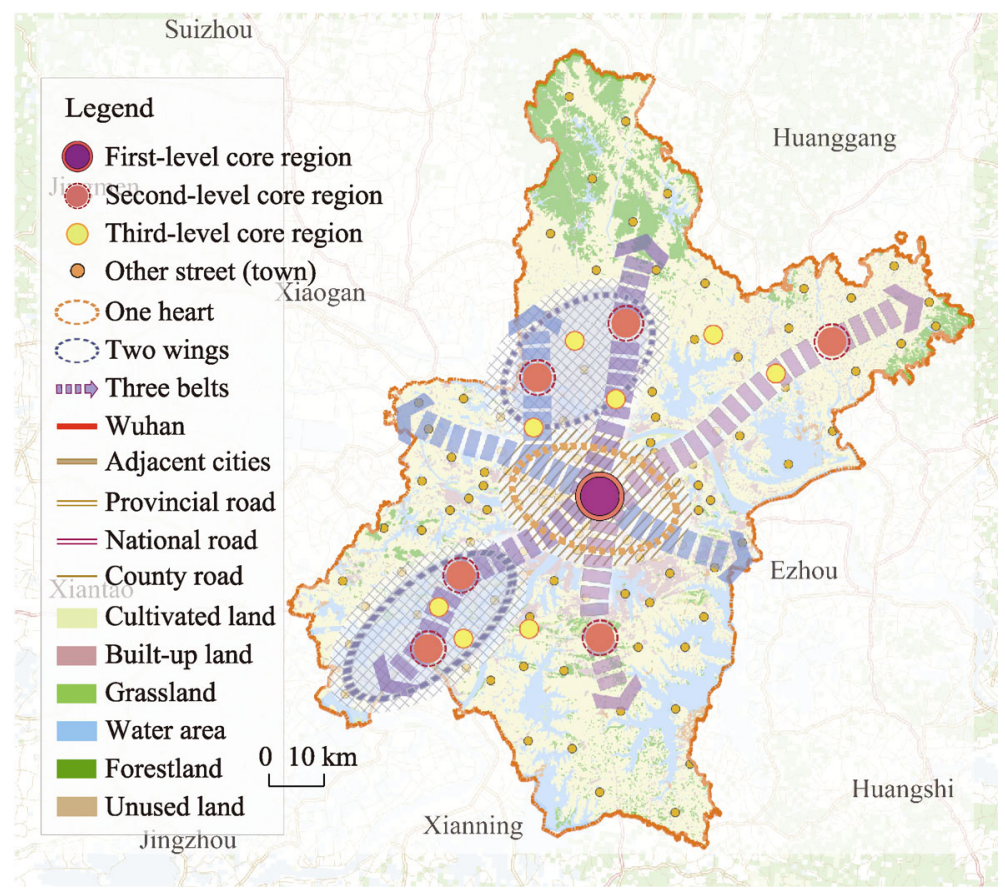

Figure 5 The patterns of spatial development and protection in Wuhan

According to the results of identification of the core areas and characterization of the development axes, the spatial development framework of "one heart, two wings, and three belts" was conceived. "One heart" refers to core area 4 with central urban areas as the main body. As the region of the aggregation of spatial elements and strategic pivot of spatial development, it undertook regional political, cultural, and economic functions. "Two wings" refers to the development groups of the northwestern in Qianchuan and Tianhe, and in the southwest in the Caidian Economic Development Zone and Dengnan. "Three belts" refers to the axes of development of Huangpi-Jiangxia, Xinzhou-Caidian, and Dongxihu-Optics Valley. Given this spatial development and protection framework of "one heart, two wings, and three belts," imbalance in spatial development can be recognized. Preferential policies should be formulated for the core areas to promote the formation of a regional development model "from point to region."

\section{Conclusions and discussion}

\subsection{Conclusions}

Patterns of development and protection form the scientific basis for a feasible layout of spaces for production, living, and ecology as well as the theoretical basis for the overall coordination of their economic, social, and ecological benefits. Based on basic geographic information and data from statistical yearbooks, we used circuit theory and the MCR in this paper to construct a pattern for the development and protection of Wuhan, and formulated a 
comprehensive expression for the spatial structure of core areas-resistance surface-development axes. The results show the following: First, the population density, GDP density, access for traffic, and rate of land urbanization were used to identify seven core area of development that consisted of 111 streets and accounted for about $20 \%$ of the total area of Wuhan. The distribution of the core areas exhibited a spatial distribution of "circular satellites," and distributions in mosaic form were highly consistent with the administrative center. Second, the resistance surface, which was extracted by determining the importance of ecosystem services, ecological sensitivity, land use type, and terrain slope, was mainly distributed in ecological spaces that offered large ecological benefits, such as the central regions of Huangpi and Xinzhou, and southwest Caidian. Areas with low and high values of resistance to spatial development in Wuhan accounted for $6.64 \%$ and $12.53 \%$ of the city's total area, respectively. Third, based on the least-cost path and current density, nine spatial development axes and eight strategic points were identified, and a channel for elements flow centered on core area 4 was formed. The area of the zone where construction was emphasized was $467.29 \mathrm{~km}^{2}$. The inconsistency between the development axis and distribution of current density was revealed, and the problem of arriving at incorrect conclusions by setting the width of the development axis subjectively was avoided. Finally, we constructed the pattern of the spatial development and protection of Wuhan using the idea of "one heart, two wings, and three belts", and recommended that the government formulate preferential policies for strategic areas to form a regional development mode "from point to region" in the new era.

\subsection{Discussion}

Research on constructing patterns of ecological security featuring development and protection axes are common, but spatial contradictions have been noted in such analyses when a non-uniform framework is used. On the basis of the summary and conclusion of the characteristics of spatial structure under the field of "function and system" in geography, a basic understanding of the "point-axis-area" spatial structure was provided. "Point" is the source of power driving the spatial elements to flow. "Axis" is the carrier of elements that connect and interact with the each other at "points," and the points and axes work together on geographic entities to form patterns of development and protection. At the same time, the development axes based on the resistance surface can guide the layout of spatial elements, arrange the order of spatial development, and minimize the impacts of spatial development-related activities on functions of ecosystem services. Therefore, by analyzing the characteristics of the spatiotemporal distribution of elements of the nature-society-economy system and the spatial structure of the "point-axis-area," patterns for the spatial development and protection of core areas were constructed by using the circuit theory model and MCR as a useful complement to prevalent research on spatial structure. Through the comparative analysis with the current development situation of Wuhan, the seven core areas, nine development axes and eight strategic points identified in this study are highly consistent with the trend of spatial development and protection in Wuhan, which shows that the research results are consistent with the objective facts. For example, the flow of spatial elements in Hankou is significantly higher than that in Wuchang, and the streets on the axis connecting core area 1 and core area 4 are developing rapidly.

However, there are still some limitations to this study: First, due to the unavailability of data, the spatiotemporal resolution of the smallest research unit used in this study was at the 
sub-district level, and thus failed to break through the administrative boundary, identify core areas, characterize the development axes, and construct patterns of development and protection at a high resolution. In future work, we plan to introduce datasets consisting of streaming data, urban POI data, and night-light data to research high-resolution geographical patterns. Second, the basis of the patterns of spatial development and protection is spatial planning given the requirement of ecological civilization construction based on the heterogeneous characteristics of the spatial elements. However, the heterogeneity and homogeneity of geographic elements have prominent scale effects. Thus, studying the construction of spatial patterns at different scales can provide comprehensive guidance for formulating policies for regional development. Third, the research on the construction of the spatial pattern based on the existing basic data reflects the comparative advantages of the spatial elements under historical accumulation, and the identified spatial pattern is the heritage and continuation of the existing space development and protection. But on the summary of the existing space development laws, there is a risk of continuing the early blind development and protection. It's necessary to further study the inherent differences in the flow patterns of elements under different scenarios in the space development trade-off as a reference and choice of space development and protection solutions for policy makers and planners. Finally, policy orientation as an important factor in the ultimate blueprint for development and the path of implementation were not adequately considered in the theoretical exploration and practical argument. Territorial space-related policies with a single development goal, with characteristics of local rather than global optimality, struggle to meet regional developmental needs that are multi-objective, multi-path, and multi-concept. Thus, guiding the transition of policy from local to global optimization should be the focus of scientific research on patterns of spatial development and protection.

\section{References}

Avon C, Bergès L, 2016. Prioritization of habitat patches for landscape connectivity conservation differs between least-cost and resistance distances. Landscape Ecology, 31(7): 1551-1565.

Azaele S, Maritan A, Cornell S J et al., 2015. Towards a unified descriptive theory for spatial ecology: Predicting biodiversity patterns across spatial scales. Methods in Ecology and Evolution, 6: 324-332.

Blatti C, Sinha S, 2016. Characterizing gene sets using discriminative random walks with restart on heterogeneous biological networks. Bioinformatics, 32(14): 2167-2175.

Blazquez-Cabrera S, Gastón A, Beier P et al., 2016. Influence of separating home range and dispersal movements on characterizing corridors and effective distances. Landscape Ecology, 31(10): 2355-2366.

Bradburd G S, Ralph P L, Coop G M, 2016. A spatial framework for understanding population structure and admixture. PLoS Genetics, 12(1): e1005703.

Carroll C, McRae B H, Brookes A, 2012. Use of linkage mapping and centrality analysis across habitat gradients to conserve connectivity of gray wolf populations in western North America. Conservation Biology, 26(1): 78-87.

Chen M X, Liu W D, Lu D D et al., 2018. Progress of China's new-type urbanization construction since 2014: A preliminary assessment. Cities, 78: 180-193.

Correa Ayram C A, Mendoza M E, Etter A et al., 2016. Habitat connectivity in biodiversity conservation: A review of recent studies and applications. Progress in Physical Geography, 40(1): 7-37.

Costanza J K, Terando A J, 2019. Landscape connectivity planning for adaptation to future climate and land-use change. Current Landscape Ecology Reports, 4(1):1-13.

Dobrynin A A, Kochetova A A, 1994. Degree distance of a graph: A degree analogue of the Wiener index. Journal of Chemical Information and Computer Sciences, 34(5): 1082-1086.

Fan J, 2018. "Territorial system of human-environment interaction": A theoretical cornerstone for comprehensive research on formation and evolution of the geographical pattern. Acta Geographica Sinica, 73(4): 597-607. (in Chinese)

Ferretti V, Pomarico S, 2013. An integrated approach for studying the land suitability for ecological corridors 
through spatial multicriteria evaluations. Environment, Development and Sustainability, 15(3): 859-885.

Huang Q, Zeng Y, Jiang Q, 2015. Progress and prospect of the study on "making great efforts to promote ecological civilization construction". China Population, Resources and Environment, 25(2): 111-120. (in Chinese)

Jin G, Chen K, Wang P et al., 2019. Trade-offs in land-use competition and sustainable land development in the North China Plain. Technological Forecasting and Social Change, 141: 36-46.

Jin G, Deng X Z, Chu X et al., 2017. Optimization of land-use management for ecosystem service improvement: A review. Physics and Chemistry of the Earth, 101: 70-77.

Jin G, Deng X Z, Zhao X D et al., 2018. Spatiotemporal patterns in urbanization efficiency within the Yangtze River Economic Belt between 2005 and 2014. Journal of Geographical Sciences, 28(8): 1113-1126.

Jin G, Li Z H, Deng X Z et al., 2019. An analysis of spatiotemporal patterns in Chinese agricultural productivity between 2004 and 2014. Ecological Indicators, 105: 591-600.

Klein D J, Randić M, 1993. Resistance distance. Journal of Mathematical Chemistry, 12(1): 81-95.

Kupfer J A, 2012. Landscape ecology and biogeography: Rethinking landscape metrics in a post-FRAGSTATS landscape. Progress in Physical Geography, 36(3): 400-420.

Leonard P B, Duffy E B, Baldwin R F et al., 2017. Gflow: Software for modelling circuit theory-based connectivity at any scale. Methods in Ecology and Evolution, 8(4): 519-526.

Li S C, Bing Z L, Jin G, 2019. Spatially explicit mapping of soil conservation service in monetary units due to land use/cover change for the Three Gorges Reservoir Area, China. Remote Sensing, 11(4): 468.

Liu H T, Tian L L, Tian Y et al., 2015. Exploring the spatial expression and governance policies of urban sprawl in Wuhan. Economic Geography, 35(4): 47-53. (in Chinese)

Luo F H, Liu Y X, Peng J et al., 2018. Assessing urban landscape ecological risk through an adaptive cycle framework. Landscape and Urban Planning, 180: 125-134.

Martin D M, Mazzotta M, Bousquin J, 2018. Combining ecosystem services assessment with structured decision making to support ecological restoration planning. Environmental Management, 62(3): 608-618.

McRae B H, Beier P, 2007. Circuit theory predicts gene flow in plant and animal populations. Proceedings of the National Academy of Sciences, 104(50): 19885-19890.

McRae B H, Dickson B G, Keitt T H et al., 2008. Using circuit theory to model connectivity in ecology, evolution and conservation. Ecology, 89(10): 2712-2724.

Nunes D M, Tomé A, Pinheiro M D, 2019. Urban-centric resilience in search of theoretical stabilisation? A phased thematic and conceptual review. Journal of Environmental Management, 230: 282-292.

Peng J, Li H L, Liu Y X et al., 2018. Identification and optimization of ecological security pattern in Xiong'an New Area. Acta Geographica Sinica, 73(4): 701-710. (in Chinese)

Peng J, Liu Y X, Wu J S et al., 2015. Linking ecosystem services and landscape patterns to assess urban ecosystem health: A case study in Shenzhen City, China. Landscape and Urban Planning, 143: 56-68.

Peng J, Yang Y, Liu Y X et al., 2018. Linking ecosystem services and circuit theory to identify ecological security patterns. Science of the Total Environment, 644: 781-790.

Roopnarine P D, Byars G, Fitzgerald P, 1999. Anagenetic evolution, stratophenetic patterns, and random walk models. Paleobiology, 25(1): 41-57.

Spear S F, Balkenhol N, Fortin M J et al., 2010. Use of resistance surfaces for landscape genetic studies: Considerations for parameterization and analysis. Molecular Ecology, 19: 3576-3591.

Thulasiraman K, Yadav M, Naik K, 2019. Network science meets circuit theory: Resistance distance, Kirchhoff index, and Foster's theorems with generalizations and unification. IEEE Transactions on Circuits and Systems I: Regular Papers, 66(3): 1090-1103.

Todes A, 2017. Shaping peripheral growth? Strategic spatial planning in a South African city-region. Habitat International, 67: 129-136.

Vasanen A, 2012. Functional polycentricity: Examining metropolitan spatial structure through the connectivity of urban sub-centres. Urban Studies, 49(16): 3627-3644.

Veneri P, 2013. The identification of sub-centres in two Italian metropolitan areas: A functional approach. Cities, 31: 177-185.

Yang J X, Gong J, Gao J et al., 2019. Stationary and systematic characteristics of land use and land cover change in the national central cities of China using intensity analysis: A case study of Wuhan City. Resources Science, 41(4): 701-716. (in Chinese)

Yu B L, Shu S, Liu H X et al., 2014. Object-based spatial cluster analysis of urban landscape pattern using nighttime light satellite images: A case study of China. International Journal of Geographical Information Science, 28(11): 2328-2355.

Zhou D, Xu J C, Wang L et al., 2015. Assessing urbanization quality using structure and function analyses: A case study of the urban agglomeration around Hangzhou Bay (UAHB), China. Habitat International, 49: 165-176. 